\title{
Prevalence of plasmodium, leptospira and rickettsia species in Northern Tanzania: a community based survey
}

\author{
Jaffu O Chilongola ${ }^{1,2}$, Elias J Sabuni ${ }^{3}$, Eliakimu Paul Kapyolo ${ }^{4}$
}

1. Kilimanjaro Christian Medical University College, P.O. Box 2240, Moshi Tanzania.

2. Kilimanjaro Clinical Research Institute, P.O. Box 2236, Moshi Tanzania.

3. Mawenzi Regional Referral Hospital, P.O. Box 3054, Moshi Tanzania.

4. Kibong'oto Infectious Diseases Hospital, P.O. Box 12, Sanya Juu, Kilimanjaro.

\section{Emails:}

j.chilongola@kcri.ac.tz; eliassabuni@gmail.com, dell.pmgabe@yahoo.com

\begin{abstract}
Background: The overlap of symptoms, geographic and seasonal co-occurrence of Plasmodium, Leptospira and Rickettsia infections makes malaria diagnosis difficult, increasing the chances of misdiagnosis. The paucity of data on the prevalence Plasmodium, Leptospira and Rickettsia infections contributes to an overly diagnosis of malaria. We aimed to determine the prevalence and distribution of Plasmodium, Leptospira and Rickettsia infections in northern Tanzania.

Methods: A community based, cross sectional survey was conducted in two sites in Northern Tanzania. PCR was used to detect Plasmodium, Leptospira and Rickettsia infections.

Results: The prevalence of P. falciparum and Leptospira spp were 31/128 (24.2\%) and 3/128 (2.3\%), respectively. No Rickettsia infection was detected in any of the two sites. Taking study sites separately, Plasmodium infection was detected in 31/63(49.2\%) of participants in Bondo while Leptospira infection was detected in 3/65(4.6\%) of participants in Magugu. Plasmodium was not detected in Magugu while no Leptospira infections were detected in Bondo. Fever was significantly associated with Plasmodium infection $(\chi 2=12.44, \mathrm{p}<0.001)$ and age $(\chi 2=17.44, \mathrm{p}=0.000)$.

Conclusion: Results from this study indicate Plasmodium infection as the main cause of fever in the studied sites. While Plasmodium and Leptospira contribute to fevers, Rickettsia infection is an insignificant cause of fever in Northern Tanzania.
\end{abstract}

Keywords: Neglected Infectious Diseases, Plasmodium, Leptospira, Rickettsia, co-occurrence, Tanzania.

DOI: https://dx.doi.org/10.4314/ahs.v20i1.25

Cite as: Chilongola JO, Sabuni EJ, Kapyolo EP. Prevalence of plasmodium, leptospira and rickettsia species in Northern Tanzania: a community based survey. Afri Health Sci. 2020;20(1):199-207. https:/ / dx.doi.org/10.4314/ahs.v20i1.25

\section{Background}

Malaria incidence and mortality rates are reported to decrease worldwide. According to the World Health Organization (WHO), between 2000 and 2015, malaria incidence rates decreased by $37 \%$ globally, and by $42 \%$ in Africa. In the same period, malaria mortality rates declined by $60 \%$

\section{Corresponding author: \\ Jaffu O Chilongola, \\ Kilimanjaro Christian \\ Medical University College, \\ P. O. Box 2240 and \\ Kilimanjaro Clinical Research Institute, \\ P. O. Box 2236, Moshi, Tanzania; \\ Email: j.chilongola@kcri.ac.tz}

globally and by $66 \%$ in the African Region. ${ }^{1}$ Despite the decrease of malaria, higher numbers of fever cases have continued to be reported. ${ }^{2-4}$ A previous study conducted in the Northern part of Tanzania reported that, out of the 870 fever cases studied, 528 (60.7\%) of clinical malaria diagnosis, malaria was the actual cause of fever in only $1.6 \%$ of the fever cases. By contrast, $40(33.9 \%)$ had leptospirosis and $36(30.5 \%)$ and $2(1.8 \%)$ of the fever cases had spotted fever group rickettsioses and typhus group rickettsioses, respectively. ${ }^{5}$ This implies the presence of alternative causes of febrile illnesses including emerging and re-emerging infectious diseases including Leptospira and Rickettsia infections ${ }^{6,7 ; 5}$. Unfortunately, apart from being known as a significant cause of non malarial Febrile Illnesses (NMFIs). ${ }^{5}$, both of these infections are also known to share similar symptoms. They also display

(C) 2020 Chilongola JO et al. Licensee African Health Sciences. This is an Open Access article distributed under the terms of the Creative commons Attribution License (https://creativecommons.org/licenses/BY/4.0), which permits unrestricted use, distribution, and reproduction in any medium, provided the original work is properly cited. 
an overlap with malaria in a terms of geographic and seasonal distribution ${ }^{8}$. For instance, the cardinal symptoms of malaria are similar to those of Leptospira and Rickettsia infections usually presenting as fever, myalgia and retro-orbital pain.

In normal practice, in areas where malaria is endemic, these symptoms are attributed to malaria and usually treated immediately out of fear of missing $P$. falciparum infection $^{9-11}$. The overlap of symptoms of infections by Plasmodium, Leptospira and Rickettsia poses a diagnosis challenge, creating the potential risks of misdiagnosis of malaria ${ }^{6 ; 11 ; 12}$. Usually this leads to overly diagnosis of malaria with significant under estimation of the burden of other causes of fevers including Leptospira and Rickettsia ${ }^{12 ; 13}$.

Furthermore, the geographic and seasonal co-occurrence of these infections and their zoonotic nature, complicate the epidemiology of the diseases in areas where they occur $^{14}$. Despite of diagnostic and therapeutic challenges posed by these diseases, the lack of knowledge of their existence may contribute to an overly diagnosis of malaria. Therefore, this study was designed to determine the prevalence of Plasmodium spp., the most common cause of fever, but also the prevalence and distribution of other causes of fever; Leptospira and Rickettsia spp. infections.

\section{Methods}

\section{Study design, sites and population}

This was a cross sectional community based pilot study that aimed to determine the prevalence of Plasmodium spp and Leptospira and Rickettsia spp. in two sites in northern Tanzania. The study was conducted from April to May, 2016. The study included 128 participants from the two sites. Study sites were chosen conveniently. Participants from the two sites were selected randomly, but to represent an approximately equal number of participants from each site. Study participants included in the study were those who had lived in the study sites for at least six months prior to the study and aged one year and above. This information was obtained through interviews with prospective participants before random sampling was performed. Children with severe illnesses including malaria were excluded from the study. The two sites were selected based on their different locations, climates and malaria transmission intensities. Magugu site is a low malaria transmission area with an estimated malaria prevalence of $1 \%{ }^{15}$. Magugu is located at $4^{\circ} 12^{\prime}$ South latitude, $35^{\circ} 45^{\prime}$ East longitude about 1392 meters above sea level. Bondo site is about 309 meters above sea level at $5^{\circ} 22^{\prime} 60^{\prime \prime}$ North and $38^{\circ} 34^{\prime} 60^{\prime \prime}$ East, with a perennial malaria transmission with a prevalence of $20.5 \%{ }^{16}$. The natives of both areas are agro-pastoralists with a moderate human-animal interaction. Both areas have two rainy seasons per year; the long rainy season between February and May and the short rains between October and December of each year. The long rainy seasons are usually followed by high numbers of reported fever cases.

\section{Sample size and Sample size estimation}

The minimum sample size for prevalence determination was estimated using the Epi Tools online sample size calculator $[Z 2 \bullet(p) \bullet(1-p)] / c 2$, where $Z=1.96$ for $95 \%$ confidence level (CI), $\mathrm{p}=$ expected true proportion of $9.0 \%$ and $\mathrm{c}=$ (minimal tolerable error at 95\% CI (0.05). Computing with the above formula gives a minimum sample size of 126.

\section{Demographic data}

Demographic data of all study participants were obtained using a structured questionnaire through face to face interview. The questionnaire used in this study was developed by a team of investigators in this study. It was first piloted on ten respondents before the actual study and these respondents were excluded during actual data collection and analysis. Validity and reliability were determined by using computer software IBM SPSS Version 20. After the pre-test, necessary adjustments in phrasing were made in the questionnaire.

\section{Blood sample collection and processing}

For each consenting participant, at least $1.5 \mathrm{~mL}$ of venous blood was collected into sterile eppendorf tubes by trained laboratory personnel using a sterile disposable syringe. At least 20ul of each sample was used for malaria diagnosis by preparation of thick and thin blood smears for malarial microscopy. ${ }^{16}$ For the sake of clinical care, about 10 ul of whole blood was subjected to malaria rapidiagnostic test (mRDT) (SD BIOLINE® Malaria Ag P. f/Pan) for malaria diagnosis. Children under the age of 5 who were found to be malaria positive by mRDT were immediately treated with Arthemether-Lumefantrine 
(ALu), the first-line antimalarial drug in Tanzania according to the national and WHO guidelines for treating uncomplicated malaria. Whole blood samples were stored at $4^{\circ} \mathrm{C}$ until DNA extraction was performed for polymerase chain reaction (PCR) testing.

\section{DNA extraction}

DNA purification was done by using QIAamp DNA Mini Kits as described elsewhere. ${ }^{17}$ An aliqout of $200 \mu \mathrm{L}$ from blood sample was lysed by QIAGEN protease and bound to QIAamp membrane by centrifugation as described by manufacturer's instructions (Fast Track Diagnostics (FTD), Luxembourg, Germany). The wash buffers AW1 and AW2, each at a time, remove residual contaminants in order to improve the purity of the DNA. Purified DNA was then eluted from QIAamp membrane by Buffer AE and stored at $-20^{\circ} \mathrm{C}$ ready for PCR assay.

\section{Detection of plasmodium, leptospira and rickettsia}

The PCR assay was carried out in an Applied Biosystems ${ }^{\circledR}$ ViiA ${ }^{\text {TM }} 7$ Real-Time PCR (Life Technologies Corporation, CA, USA). The Mastermix Kit (Fast-Track Diagnostics ${ }^{\circledR}$ (FTD), Luxembourg) for Tropical Fever Core was used to prepare the reaction mix. For single reaction, $12.5 \mu \mathrm{l}$ of FTD buffer, $1.5 \mu \mathrm{l}$ of Tropical Fever primers and probe mix (TF PP mix) and $1 \mu$ l Enzyme mix were placed into a single MicroAmp ${ }^{\circledR}$ Optical 8-Tube compatible with the ViiA ${ }^{\mathrm{TM}} 7$ RT PCR, followed by $10 \mu \mathrm{l}$ of sample. The same was done for all samples, positive control and extracted negative control. The detection of pathogens were done at wavelengths of $520 \mathrm{~nm}$ for Plasmodium (TF2 PP), 610nm for Leptospira (TF2 PP) and 550nm for Rickettsia (TF1 PP). The positive, negative and internal control used in this assay were commercially prepared by Fast-Track Diagnostics (FTD), Luxembourg Germany). The positive control contained plasmids for the detection of Plasmodium, Leptospira and Rickettsia spp., negative control contained lysis buffer while the internal control contained Streptococcus equi (Sequi) which was also used as an extraction control. The PCR was set for 40 cycles. At the end of the run, amplification plots were reviewed in order to adjust the threshold line above all the background noise as instructed by the man- ufacturer. A positive result was assigned if the threshold cycle $(\mathrm{Ct})$ value was $\leq 30$ cycles for all pathogens.

\section{Data processing and statistical analysis}

Data was analyzed using Stata v.14 (College Station, Texas 77845 USA). Data was characterized into demographic (age, sex, residence) and clinical (body temperature and prevalence of Plasmodium, Leptospira and Rickettsia infections). Age of participants was categorized into three groups of $<5,5-15$ and above 15 years. Descriptive statistics were used to summarize demographic and clinical characteristics of study participants. Chi-square $(\chi 2)$ test was used to determine associations between categorical data on demographic and clinical characteristics, in order to establish the extent of co-infections with studied pathogens and the most important cause of fever. Fisher's exact test was used in cases when expected counts were less than 5. A two tailed $p$ value of 0.05 or less was considered the cut-off for statistical significance.

\section{Ethical issues}

This study was conducted after ethical approval by the Kilimanjaro Christian Medical University College (KCMUCo) Research and Ethics Committee (Certificate \#995). Informed consent was obtained from all study participants after explanations were made regarding the study in the native language of the participants. For participants below 18 years of age, consent was obtained from their parents and/or guardians. No personal identifiers were disclosed in any form or stage of the study including reports.

\section{Results}

Socio- demographic and clinical characteristics of study participants

Table 1 presents descriptive statistics of demographic and clinical characteristics of the study participants. Both study sites had almost equal number of study participants. Out of the 128 participants, $64.8 \%$ were above 15 years old while $16.4 \%$ among them were children below five years of age. Nearly two-thirds or $66.4 \%$ were females with only $8.1 \%$ of all participants found to have fever at the time of survey. 
Table 1 : Socio- demographic and clinical characteristics of study participants

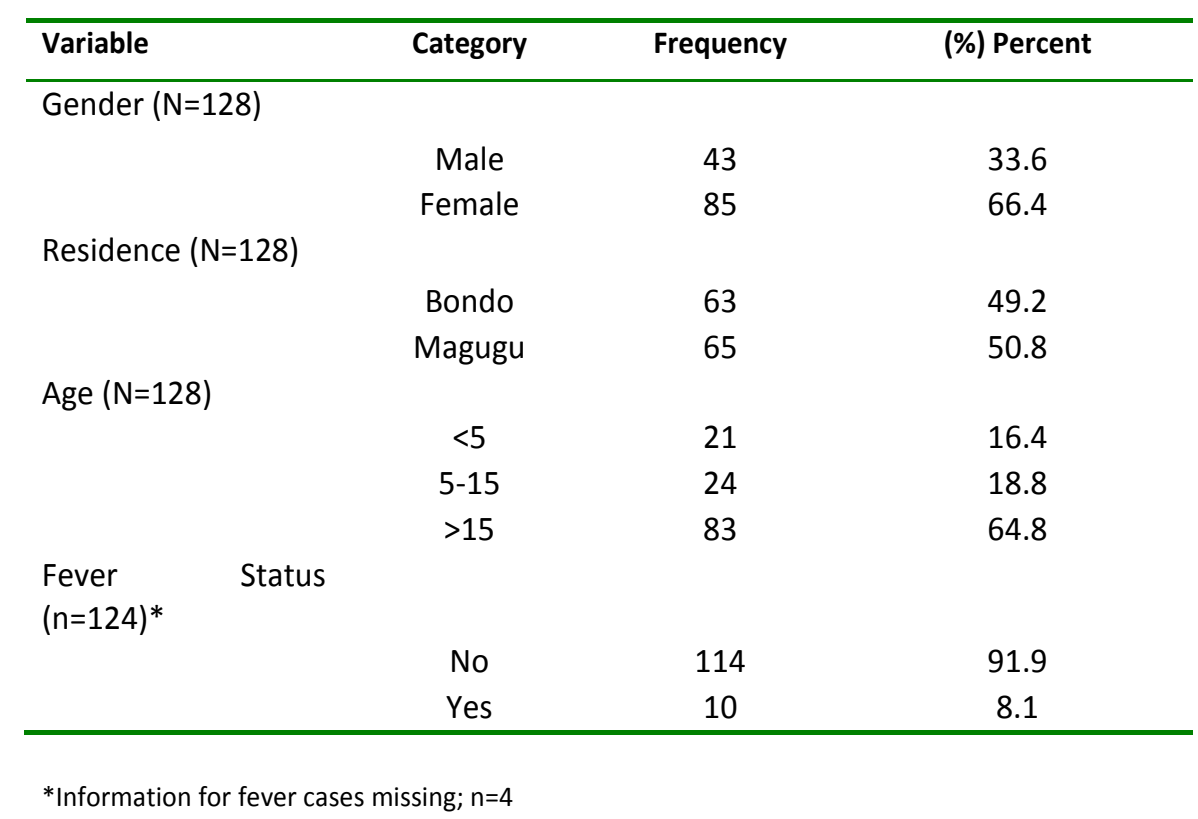

Prevalence of plasmodium, leptospira and rickettsia lence of Plasmodium, Leptospira and Rickettsia. We did not infection

In table 2 , we show the overall and site specific prevadetect any positive cases of Plasmodium were recorded in Magugu ward, no Leptospira cases in Bondo ward. We did not detect any Rickettsia infection in any of the two sites.

Table 2 : Prevalence of Plasmodium, Leptospira and Rickettsia species ( $\mathrm{N}=128)$

\begin{tabular}{lllll}
\hline Pathogen & Category & Prevalence & & \\
\cline { 3 - 4 } Plasmodium & & Bondo $\mathrm{n}=63(\%)$ & Magugu $\mathrm{n}=65(\%)$ & Overall Prevalence $\mathrm{N}=128(\%)$ \\
& & & & \\
& Negative & $32(50.8)$ & $65(100)$ & $97(75.8)$ \\
Leptospira & Positive & $31(49.2)$ & $0(0.0 \%)$ & $31(24.2)$ \\
& & & & \\
& Negative & $63(100)$ & $62(95.4)$ & $125(97.7)$ \\
Rickettsia & Positive & $0(0.0 \%)$ & $3(4.6)$ & $3(2.3)$ \\
& & & & \\
& Negative & $63(100)$ & $65(100)$ & $128(100)$ \\
& Positive & $0(0.0 \%)$ & $0(0.0 \%)$ & $0(0.0 \%)$ \\
\hline
\end{tabular}

Association of demographic factors with Plasmodium, Leptospira and Rickettsia infections

Plasmodium infection was observed to occur at a higher frequency in the age group of 5-15 years, with a high- er proportion of $66.7 \%$ in females than males. Leptospira infection was observed to occur in the age group of $>15$ years, with higher proportion $(66.7 \%)$ in male than females (Table 3). Further statistical analysis was limited due to the small number of positive cases. 
Table 3: Association of demographic factors with Plasmodium, Leptospira and Rickettsia infections

\begin{tabular}{lcccc}
\hline Variable & Category & \multicolumn{2}{c}{ Number of Cases } \\
\hline $\begin{array}{l}\text { Overall } \\
\text { prevalence } \\
\text { Gender }\end{array}$ & & $\begin{array}{c}\text { Plasmodium (\%) } \\
\text { Leptospira (\%) }\end{array}$ & $\begin{array}{c}\text { Rickettsia (\%) } \\
0(0.0 \%)\end{array}$ \\
& Male & $10(33.3 \%)$ & $2(66.7 \%)$ & $0(0.0 \%)$ \\
Residence & Female & $21(66.7 \%)$ & $1(33.3 \%)$ & $0(0.0 \%)$ \\
& Bondo & $31(100 \%)$ & $0(0.0 \%)$ & $0(0.0 \%)$ \\
Age & Magugu & $0(0.0 \%)$ & $3(100 \%)$ & $0(0.0 \%)$ \\
& $<5$ & $7(22.6 \%)$ & $0(0.0 \%)$ & $0(0.0 \%)$ \\
& $5-15$ & $13(41.9 \%)$ & $0(0.0 \%)$ & $0(0.0 \%)$ \\
& $>15$ & $11(35.5 \%)$ & $3(100 \%)$ & $0(0.0 \%)$ \\
\hline
\end{tabular}

Association of fever with demographic factors and Plasmodium, Leptospira and Rickettsia infections

Results presented in Table 4 shows that, there was a significant association between Plasmodium infection and fever $\left(\chi^{2}=12.44, \mathrm{p}<0.001\right)$ such that fever was almost exclusively attributed to Plasmodium infection. We also re- port a significant association between age of participants and fever $(\chi 2=17.44, \mathrm{p}=0.000)$ whereby the middle age group of 5-15 years old, had 6 out of the 10 fever cases recorded. All cases of fever were recorded in Bondo ward and thus an obvious strong association is reported. Besides that, Leptospira infection and gender were found to have no significant association with fever. 
Table 4 : Association of fever with demographic factors and Plasmodium, Leptospira and Rickettsia infections ( $\mathrm{N}=128$ ).

\begin{tabular}{|c|c|c|c|c|}
\hline \multirow[t]{2}{*}{ Variable } & \multicolumn{2}{|c|}{ Fever status } & \multirow{2}{*}{$\begin{array}{c}\text { Pearson } \\
\text { x-Value }\end{array}$} & \multirow{2}{*}{$\begin{array}{c}x^{2} \\
\text { P-value }\end{array}$} \\
\hline & Fever $^{a}$ & No fever & & \\
\hline \multicolumn{5}{|l|}{ Plasmodium } \\
\hline Positive & $7(23.3)$ & 23 (76.7) & & \\
\hline Negative & $3(3.2)$ & 91 (96.8) & 12.44 & $<0.001 * *$ \\
\hline \multicolumn{5}{|l|}{ Leptospira } \\
\hline Positive & $0(0.0)$ & 3 (100.0) & & \\
\hline Negative & $10(8.3)$ & $111(91.7)$ & 0.27 & 0.604 \\
\hline \multicolumn{5}{|l|}{ Rickettsia } \\
\hline Positive & 0 (0.0\%) & 0 (0.0\%) & & \\
\hline Negative & $0(0.0 \%)$ & $124(100.0)$ & $0(0.0 \%)$ & - \\
\hline \multicolumn{5}{|l|}{ Residence } \\
\hline Bondo & $10(16.1)$ & 52 (83.9) & & \\
\hline Magugu & $0(0.0)$ & $62(100.0)$ & 10.88 & - \\
\hline \multicolumn{5}{|l|}{$\mathrm{Age}^{\mathrm{b}}$} \\
\hline$<5$ & $3(15.8)$ & $16(84.2)$ & & \\
\hline $5-15$ & $6(25.0)$ & $18(75.0)$ & & \\
\hline$>15$ & $1(1.2)$ & $80(98.8)$ & 17.44 & $0.000 * *$ \\
\hline \multicolumn{5}{|l|}{ Gender } \\
\hline Male & $5(12.8)$ & $34(87.2)$ & & \\
\hline Female & $5(5.9)$ & $80(94.1)$ & 1.74 & 0.188 \\
\hline
\end{tabular}

\footnotetext{
${ }^{a}$ Fever case was defined when axillary temperature was $\geq 37.5^{\circ} \mathrm{C}$. Four missed observation on body temperature

${ }^{\mathrm{b}}$ Fishers exact test; ${ }^{* *}$ Significant at $\mathrm{p}<0.05$
}

\section{Discussion}

Diagnosing causes of acute febrile illnesses in resource poor settings such as Tanzania is one of the biggest challenges of quality health care delivery. This is because failure to make correct diagnoses impedes provision of the correct anti-microbials to patients, which would promote unnecessary antimicrobial use with consequences of development of antimicrobial resistance. The use of highly sensitive molecular tools to establish true prevalence of pathogens that cause fevers is an important step towards proper fever management and rational antimicrobial use. The current study has found the prevalence of P. falciparum infection in Bondo to be $49.2 \%$, implying about half of the sampled individuals in Bondo. This is a very high prevalence compared to data collected by previous studies. Malaria prevalance in the area has shown significant shifts in the past decade. Prevalence data collected in
2009 showed a prevalence of $32.8 \%$ in the rainy season ${ }^{18}$. This prevalence declined to about $12 \%$ in $2011^{19}$, where as the lowest prevalence of $8.6 \%$ was recorded in the year $2013^{20}$. A more recent study conducted in the study area in 2016 reported a prevalence of $20.5 \%{ }^{16}$. Although the observed fluctuations may have different interpretations, one key explanation is likely to question on the persistent efficiency of the decade long interventions to control malaria in the area.

For about a decade now, the government of Tanzania has implemented the Tanzanian National Voucher Scheme (TNVS) with a significant increase in the availability and accessibility of insecticide-treated nets (ITNs). The initiative has mainly targeted pregnant mothers and children by subsidizing costs of bed-nets ${ }^{21}$. Parallel to this, other initiatives such as the Under-five Catch-up Campaign and Universal Coverage Campaign continued to provide an 
effective integrated malaria control environment through increased ITN use, subsidized tests, artemisin-based medicines and massive community sensitization ${ }^{22}$. The net result of these strategies had been a substantial reduction in the incidence and prevalence of malaria in Tanzania as reported by recent studies ${ }^{21 ; 22}$. Our results show a sharp rise in the prevalence of malaria in the study area. Whether this is a consequence of breakdown of government inteventions or other related factors, it remains to be etsablished.

This study also found no Plasmodium infection in Magugu, which means that the prevalence might be at a very low rate, despite a favorable and suggestive climate for malaria endemicity. This result is in confluence with a previous study which also reported low prevalence of malaria in Magugu ${ }^{23}$ and with National Malaria Survey which reported prevalence of $<1 \%$ in Manyara region ${ }^{24}$. Zero prevalence of Plasmodium infection reported from Magugu by this study, can be explained from different perspectives, one of which could be the use of Magugu site as a trial site for pesticide efficacy studies. Magugu site has been used as a trial site by the Tanzania Pesticides Research Institute (TPRI) over the years. This might have kept the population of malaria vector mosquito species at a minimum, and reflected as low prevalence of malaria ${ }^{25}$.

The prevalence of Leptospira infection in Magugu alone was found to be $4.6 \%$, with no infections detected in Bondo site. This finding implies an active circulation of Leptospira spp. Although does not exclude the presence of Leptospira infections in Bondo considering the relatively small sample size included in the study. Magugu site has a richer human-animal interaction compared to Bondo site. Since transmission of Leptospira rely on conducive climate as well as presence of suitable hosts and an intimate human-animal-rodent interaction ${ }^{10 ; 26}$, it is not surprising that Leptospira infection was detected in Magugu site and not Bondo site. Although our study used sensitive tools to detect Leptospira infection, with studies that enroll large numbers of participants, more realistic data could be reported.

In this study, no case of Rickettsia infection was found either site. Rickettsia have long been known to be transmisstted by Ixodid ticks and migratory birds ${ }^{27-29}$. Although study sites involved in the present study have a reasonable interaction between humans with their domestic animals, it cannot directly be established whether they also have contact with the vector ticks and migratory birds. However, previous studies suggest the possibility of having active circulation of this infection especially in the Northern part of Tanzania. For instance, a study by Prabhu and colleagues found seroprevalence of $8.0 \%$ for spotted fever group rickettsiosis and $0.5 \%$ for typhus group rickettsiosis another study by Crump and colleagues reported seroprevalence of $30.5 \%$ for spotted fever group rickettsiosis and $1.8 \%$ for typhus group rickettsiosis ${ }^{5}$. It has to be noted that majority of these studies measured the presence of antibody to Rickettsia, falling short of the frequently reported cross reactivity of antibodies to similar pathogens. This study shows that Rickettsia infections are very rare in the study areas. We are confident that results form this study are reliable and that the negative results in samples from the sites correctly report the absence of Rickettsia infection in the studied sites.

Regarding the association between studied pathogens and fever, the current study found that nearly a quarter $(23.3 \%)$ of individuals with Plasmodium infection in Bondo, had fever. Besides that, $3.2 \%$ of those with fever were found to have neither Leptospira nor Plasmodium, which indicatesa possibilty of presence of other causes of fever with unexplained etilogy. Although it could be generalized that the remaining three quarters of Plasmodium infected but without fever were asymptomatic malaria cases, our finding does not rule out infection by other etiologies of fever not included in our tests. This argument is justified by a study previously conducted in Magugu which reported that, despite the low malaria transmission intensity in Magugu site, more than $40 \%$ of patients were provisionally misdiagnosed as malaria cases, while only $<1 \%$ of provisionally diagnosed malaria cases were confirmed as true malaria cases by microscopy ${ }^{31}$. Testing a wider range of fever causing pathogens in a pannel, could provide a refined set of findings. Furthermore, Leptospira infection and site of residence could not be associated with fever since all fevers were from Bondo site while all Leptospira cases were detected in Magugu.

We have found that the age group of between 5 and 15 had the biggest malaria infection burden. A shift in the trend of infection from the chikdren below the age of 5 years to school children aged between 6 and 15 years has recently been reported ${ }^{16}$. Since most of malaria interventions over the past decade have targeted children aged $<5$ years $^{21 ; 22}$, in a likely decreased effort in malaria control subsidies, from our findings we suggest a possible reduc- 
tion in immunity to malaria in children aged between 6 and 15 years who were the main target group for malaria control interventions when they were aged $<5$ years. Leptospira infections were observed to occur at higher frequency in males rather than females participants. We propose this observation to be a consequence of higher outdoor activities by males with increased chances of coming into contact with reservoir hosts. Our finding is congruent with previous reports ${ }^{32-34}$.

\section{Conclusion}

We report the presence of Plasmodium, Leptospira infections in northern Tanzania but not of Rickettsia spp infections. $P$. falciparum infection prevalence is higher in Bondo site than previously reported in the past ten years but no Leptospira infection was detected in Bondo. Leptospira spp was detected in Magugu only with a small proportion of infection. No Rickettsia infection was detected in any of the study sites. Most of the fevers reported in this study were attributed to P.falciparum infection. Although our study was limited by the small sample size, our findings provide a useful piece of pilot data regarding the prevalence of the three pathogens in the two sites, which may be generalized for Northern Tanzania due the distinct differences of the study sites. We recommend larger studies that test a wider range of pathogens that cause fever.

\section{Acknowledgements}

This study was financially supported by the Building Stronger Universities, phase 3 (BSU-3) initiative of the Danish International Development Agency (DANIDA). We acknowledge the technical assistance of Mr Athumani Mchana for laboratory analysis of samples; we also thank and the study participants for accepting to take part in this study. We acknowledge the logistical support provided by The Kilimanjaro Christian Medical University College and the Kilimanjaro Clinical Research Institute in the entire study.

\section{Conflict of interest}

None declared.

\section{References}

1. World Health Organization. World malaria report 2015. World Health Organization, 2016

2. Baltzell K, Elfving K, Shakely D et al. Febrile illness management in children under five years of age: a qualitative pilot study on primary health care workers $\Gamma$ ÇÖ practices in Zanzibar. Malaria Journal 2013; 12(1):37.
3. Chappuis F, Alirol E, d'Acremont V, Bottieau E, Yansouni CP. Rapid diagnostic tests for non $\Gamma$ ÇÉmalarial febrile illness in the tropics. Clinical Microbiology and Infection 2013; 19(5):422-431.

4. Chipwaza B, Mhamphi GG, Ngatunga SD et al. Prevalence of bacterial febrile illnesses in children in Kilosa district, Tanzania. PLoS Neglected Tropical Diseases 2015; 9(5):e0003750.

5. Crump JA, Morrissey AB, Nicholson WL et al. Etiology of severe non-malaria febrile illness in Northern Tanzania: a prospective cohort study. PLOS Neglected Tropical Diseases 2013; 7(7):e2324.

6. Garc $+\lceil a-R u+i z$ D, Mart+¡nez-Guzm+ín MA, C+írdenas-Vargas A et al. Detection of dengue, west Nile virus, rickettsiosis and leptospirosis by a new real-time PCR strategy. Springer Plus 2016; 5(1):1-9.

7. Thompson CN, Blacksell SD, Paris DH et al. Undifferentiated febrile illness in Kathmandu, Nepal. The American Journal of Tropical Medicine and Hygiene 2015; 92(4):875-878. 8. Mediannikov O, Socolovschi C, Edouard S et al. Common epidemiology of Rickettsia felis infection and malaria, Africa. Emerging Infectious Diseases 2013; $19(11): 1775$. 9. Biggs HM, Hertz JT, Munishi OM et al. Estimating leptospirosis incidence using hospital-based surveillance and a population-based health care utilization survey in Tanzania. PLoS Neglected Tropical Diseases 2013; 7(12):e2589.

10. de Vries SG, Visser BJ, Nagel IM et al. Leptospirosis in Sub-Saharan Africa: a systematic review. International Journal of Infectious Diseases 2014; 28:47-64.

11. Punjabi NH, Taylor WR, Murphy GS et al. Etiology of acute, non-malaria, febrile illnesses in Jayapura, northeastern Papua, Indonesia. The American Journal of Tropical Medicine and Hygiene 2012; 86(1):46-51.

12. Torgerson PR, Hagan JE, Costa F et al. Global burden of leptospirosis: estimated in terms of disability adjusted life years. PLoS Neglected Tropical Diseases 2015; 9(10):e0004122.

13. Chikeka I, Dumler JS. Neglected bacterial zoonoses. Clinical Microbiology and Infection 2015; 21(5):404-415.

14. Socolovschi C, Pages Fdr, Ndiath MO, Ratmanov P, Raoult D. Rickettsia species in African Anopheles mosquitoes. PloS One 2012; 7(10):e48254.

15. Mwanziva CE, Kitau J, Tungu PK et al. Transmission intensity and malaria vector population structure in Magugu, Babati District in northern Tanzania. Tanzania Journal of Health Research 2011; 13(1):54-61.

16. Athanase E, Ndaro A, Minja L, Chilongola J. Association between Malaria Prevalence and Seropositivity 
of Immunoglobulin G Subtypes Directed to Plasmodium falciparum Merozoite Surface Protein 1-19. International Journal of Tropical Disease \& Health 2016; 19(1):1-13.

17. Zavala-Castro J, Zavala-Vel+ízquez J, Walker D, $\mathrm{P}+\neg$ rez-Osorio J, Peniche-Lara G. Severe human infection with Rickettsia felis associated with hepatitis in $\mathrm{Yu}-$ catan, Mexico. International Journal of Medical Microbiology 2009; 299(7):529-533.

18. Mmbando BP, Msangeni HA, Sembuche SH et al. Epidemiology of malaria in an area prepared for clinical trials in Korogwe, north-eastern Tanzania. Malaria Journal 2009; 8(1):165.

19. Jones S, Grignard L, Nebie I et al. Naturally acquired antibody responses to recombinant Pfs 230 and Pfs48/45 transmission blocking vaccine candidates. Journal of Infection 2015; 71(1):117-127.

20. Kajeguka DC, Kaaya RD, Mwakalinga S et al. Prevalence of dengue and chikungunya virus infections in north-eastern Tanzania: a cross sectional study among participants presenting with malaria-like symptoms. BMC Infectious Diseases 2016; 16(1):183.

21. Renggli S, Mandike R, Kramer K et al. Design, implementation and evaluation of a national campaign to deliver 18 million free long-lasting insecticidal nets to uncovered sleeping spaces in Tanzania. Malaria Journal 2013; 12(1):85.

22. Bonner K, Mwita A, McElroy PD et al. Design, implementation and evaluation of a national campaign to distribute nine million free LLINs to children under five years of age in Tanzania. Malaria Journal 2011; 10(1):73.

23. Mwanziva C, Manjurano A, Mbugi E et al. Defining malaria burden from morbidity and mortality records, self treatment practices and serological data in Magugu, Babati District, northern Tanzania. Tanzania Journal of Health Research 2011; 13(2):93-96.

24. TACAIDS ZAC, NBS OCGS ICF. Tanzania HIV/ AIDS and Malaria Indicator Survey 2011 ГÇô12. Dar es Salaam, Tanzania Dar es Salaam, Tanzania: Tanzania Commission for AIDS (TACAIDS), Zanzibar AIDS Commission (ZAC), National Bureau of Statistics (NBS), Office of the Chief Government Statistician (OCGS), and ICF International 2013.
25. Msangi S, Lawrence B, Masenga C. Comparative Effectiveness of Neonicotinoid (Thiamethoxam) and Organophosphate (Azamethiphos) Against Synanthropic House Flies. Editorial Advisory Board e 2005; 15(4):493-497. 26. Maze MJ, Biggs HM, Rubach MP et al. Comparison of the Estimated Incidence of Acute Leptospirosis in the Kilimanjaro Region of Tanzania between 2007 $\Gamma$ Çô08 and 2012ГÇô14. PLoS Neglected Tropical Diseases 2016; 10(12):e0005165.

27. Matsumoto K, Ogawa M, Brouqui P, Raoult D, Parola P. Transmission of Rickettsia massiliae in the tick, Rhipicephalus turanicus. Medical and Veterinary Entomology 2005; 19(3):263-270.

28. Hildebrandt A, Franke J, Meier F et al. The potential role of migratory birds in transmission cycles of Babesia spp., Anaplasma phagocytophilum, and Rickettsia spp. Ticks and Tick-borne Diseases 2010; 1(2):105-107.

29. Souza CE, Moraes-Filho J, Ogrzewalska M et al. Experimental infection of capybaras Hydrochoerus hydrochaeris by Rickettsia rickettsii and evaluation of the transmission of the infection to ticks Amblyomma cajennense. Veterinary Parasitology 2009; 161(1):116-121.

30. Prabhu M, Nicholson WL, Roche AJ et al. Q fever, spotted fever group, and typhus group rickettsioses among hospitalized febrile patients in northern Tanzania. Clinical Infectious Diseases 2011; 53(4):e8-e15.

31. Mwanziva C, Shekalaghe S, Ndaro A et al. Overuse of artemisinin-combination therapy in Mto wa Mbu (river of mosquitoes), an area misinterpreted as high endemic for malaria. Malaria Journal 2008; 7(1):232.

32. Felzemburgh RD, Ribeiro GS, Costa F et al. Prospective study of leptospirosis transmission in an urban slum community: role of poor environment in repeated exposures to the Leptospira agent. PLoS Neglected Tropical Diseases 2014; 8(5):e2927.

33. Hagan JE, Moraga P, Costa F et al. Spatiotemporal determinants of urban leptospirosis transmission: fouryear prospective cohort study of slum residents in Brazil. PLoS Neglected Tropical Diseases 2016; 10(1):e0004275.

34. Reis RB, Ribeiro GS, Felzemburgh RD et al. Impact of environment and social gradient on Leptospira infection in urban slums. PLoS Neglected Tropical Diseases 2008; 2(4):e228. 